\title{
Um índice de capacidade para especificações unilaterais
}

\author{
Gladys D. Cacsire Barriga \\ Dra. Eng. Produção PRO - POLI - USP \\ Linda Lee Ho - EP-USP \\ Profa. Dra. PRO - POLI - USP \\ Caixa Postal 61548, 05424-970 \\ Wagner Sousa Borges - IME USP \\ Prof. Dr IME-USP \\ Caixa Postal 66281, 05315-970
}

\begin{abstract}
Resumo
Nos últimos anos, índices de capacidade de processos têm sido usados freqüentemente para determinar se um processo é capaz de produzir itens em conformidade com a tolerância especificada. No entanto, poucos estudos têm sido feitos acerca de índices de capacidade para situações em que há apenas um limite de especificação. Neste trabalho, um índice para situações em que há apenas um limite de especificação é apresentado. Ele é invariante com respeito à fração não-conforme do processo e "calibrado" pelo índice $C_{p k}$. São desenvolvidos também aspectos inferenciais, do ponto de vista bayesiano, para amostragem binomial.
\end{abstract}

\section{Palavras-chave}

Índices de capacidade, fração não-conforme, especificação unilateral, estatística bayesiana.

\section{Process capability index for one-sided specification limit}

\begin{abstract}
Process capability indices have become popular tools to describe how well a process can meet specified tolerances. However, little attention has been given to the study of these indices when the specified tolerances are one-sided. In this work we present an index for such situations. The proposed index is invariant with respect to process fraction non-conforming and it is " $C_{p k}$ calibrated". Bayesian inference procedure under a binomial sampling is also developed and described for the proposed index.
\end{abstract}

\section{Key words}

Process capability indices; Non-conforming fraction; One-sided specification limit; Bayesian analysis. 


\section{INTRODUĈ̣̃O}

As indústrias, de uma maneira geral, têm optado por utilizar índices de capacidade para quantificar a fração não-conforme de processos. Neste sentido, informações são avaliadas para determinar se seus processos são capazes de gerar produtos que atendam às especificações exigidas por clientes internos ou externos. Nos últimos anos, o uso de índices de capacidade nas indústrias de manufatura tem-se intensificado, além disso, esse procedimento tem recebido uma atenção substancial na literatura estatística voltada para o controle de qualidade. Veja, por exemplo, os trabalhos de Bissel (1990), Boyles (1991), Spiring (1991), Johnson (1992), Kotz; Pearn \& Johnson (1993), Pearn; Kotz \& Johnson (1992), Pearn \& Kotz (1994), Chen (1994-95), Choi; Nam \& Park (1996), Gupta \& Kotz (1997), Pearn \& Chen (1997), Kaminsky et al. (1998), Wang \& Chen (1998-99), Bothe (1999), Wen \& Mergen (1999), Borges \& Ho (2000, 2001) e muitos outros autores. Os índices mais freqüentemente usados para avaliar a capacidade do processo são $C_{p}$ e $C_{p k}$, definidos respectivamente por:

$$
\begin{aligned}
& C_{p}=\frac{V-L}{6 \sigma} \\
& C_{p k}=\min \left(\frac{V-\mu}{3 \sigma}, \frac{\mu-L}{3 \sigma}\right)
\end{aligned}
$$

onde $V$ denota o limite superior de especificação, $L$ o limite inferior de especificação, $\mu$ a média do processo e $\sigma$ o desvio padrão do processo.

Seja $X_{1}, \ldots, X_{n}$ uma amostra aleatória do processo, os estimadores naturais destes índices são respectivamente:

$$
\begin{aligned}
& \hat{C}_{p}=\frac{V-\bar{X}}{6 s} \\
& \hat{C}_{p k}=\min \left(\frac{V-\bar{X}}{3 s}, \frac{\bar{X}-L}{3 s}\right)
\end{aligned}
$$

em que $\bar{X}=1 / n \sum X_{i}$ e $s=\left[1 /(n-1) \sum\left(X_{i}-\bar{X}\right)^{2}\right]^{0,5}$ são utilizados como estimadores de $\mu$ e $\sigma$. Propriedades das distribuições amostrais de $\hat{C}_{p}$ e $\hat{C}_{p k}$ para processos normais foram estudadas por diversos autores, tais como Kane (1986), Chou \& Owen (1989). Uma abordagem completa pode ser encontrada em Kotz \& Johnson (1993).

O índice $C_{p k}$ pode ainda ser utilizado para processos que tenham somente um limite de especificação. Para tanto, basta considerar $L \rightarrow-\infty$ ou $V \rightarrow \infty$. Se existe apenas o limite inferior de especificação, tem-se

$$
C_{p k i}=\frac{\mu-L}{3 \sigma}
$$

Da mesma forma, se existe apenas o limite superior de especificação, tem-se

$$
C_{p k s}=\frac{V-\mu}{3 \sigma}
$$

Índices de capacidade como $C_{p}, C_{p k}$ e outros, entretanto, são fortemente influenciados pela forma da distribuição da variável de interesse $X$, como ressaltam Borges \& Ho (2000, 2001). Neste trabalho, o índice de capacidade $C_{e s}$ é proposto. Tal índice é invariante com relação a mudanças na forma da distribuição de $X$ e para situações em que existe apenas um limite superior de especificação. Ele baseia-se na fração não-conforme do processo e é calibrado por $C_{p k s}$. Os resultados apresentados podem ser facilmente adaptados para situações em que existe apenas um limite inferior de especificação.

Na Seção 2, procedimento de inferência bayesiana e um teste de demonstração de capacidade para o índice proposto são desenvolvidos com base na amostragem binomial. Outras abordagens bayesianas distintas da apresentada neste trabalho podem ser encontradas em Cheng \& Spiring (1989) e Bernardo \& Irony (1996).

Considere um processo com limite superior de especificação $V$ e $X$ uma variável de interesse que obedece a uma distribuição normal com média $\mu$ e variância $\sigma^{2}$, então a fração não-conforme do processo à direita do limite superior de especificação, denotada por $\gamma_{s}$ é definida como:

$$
\gamma_{s}=1-P(X \leq V)=1-\Phi\left(\frac{V-\mu}{\sigma}\right)
$$

conseqüentemente

$$
3 C_{p k s}=\left(\frac{V-\mu}{\sigma}\right)=\Phi^{-1}\left(1-\gamma_{s}\right)
$$

Esta igualdade sugere que um índice de capacidade pode ser definido com base na fração não-conforme do processo como:

$$
C_{e s}=\left(\frac{\Phi^{-1}\left(1-\gamma_{s}\right)}{3}\right)
$$

onde $\Phi($.$) denota a função de distribuição acumulada da$ normal padrão. 
INFERÊNCIA BAYESIANA DE $C_{E S}$ COM AMOSTRAS BINOMIAIS

Seja $X_{l}, \ldots, X_{n}$ uma amostra aleatória do processo e considerem-se as variáveis aleatórias de Bernoulli definidas por:

$$
Y_{i}=\left\{\begin{array}{ccc}
1 & \text { se } & X_{i}>V \\
0, & \text { caso } & \text { contrário }
\end{array}\right.
$$

com probabilidade de sucesso $P\left(X_{i}>V\right)=\gamma_{s}$. Para desenvolver aspectos inferenciais segundo uma abordagem bayesiana, considere que a incerteza acerca de $\gamma_{s}$ seja descrita por uma distribuição a priori Beta de parâmetros $a$ e $b$. Utilizando resultados da teoria bayesiana tem-se que a distribuição a posteriori de $\gamma_{s}$ segue também uma distribuição Beta de parâmetros $(n-t+b)$ e $(a+t)$, onde $t=\sum_{i=1}^{n} Y_{i}$, sendo $n$ o tamanho da amostra. A demonstração destes resultados pode ser encontrada no Apêndice A. E utilizando uma função perda quadrática não é difícil mostrar que o estimador de Bayes de $C_{e s}$ é dado por:

$$
\hat{C}_{e s}=\frac{E\left[\Phi^{-1}(U)\right]}{3}
$$

em que $U \sim \operatorname{Beta}(n+b-t, a+t)$.(Para maiores detalhes veja Apêndice A.) Fundamentada na Lei Forte dos Grandes Números, um valor aproximado de $E\left[\Phi^{-1}(U)\right]$ em (6) pode ser obtido por integração numérica ou ainda por uma estimativa de Monte Carlo dada por:

$$
\hat{E}\left[\Phi^{-1}(U)\right] \cong 1 / m \sum_{i=1}^{m} \Phi^{-1}\left(U_{i}\right)
$$

em que $U_{1}, \ldots, U_{m}$ é uma amostra aleatória suficientemente grande da distribuição $\operatorname{Beta}(n+b-t, a+t)$. Esta possibilidade constitui uma alternativa atraente em vista dos recursos computacionais hoje disponíveis. Veja isto através de um exemplo numérico.

Exemplo - Num processo de usinagem, uma parada de máquina é geralmente um evento crítico do ponto de vista da produtividade. $\mathrm{O}$ processo é considerado adequado se o número de paradas de máquina em cada semana for no máximo quatro (limite superior de especificação para $N$ paradas de máquina). Para monitorar esta variável suponha que uma carta de controle $C$ tenha sido utilizada e em condição de controle, $N$ tem distribuição do Poisson com média 1. Na Tabela 1 estão resumidos os resultados de 36 semanas coletados nesta condição.

De acordo com a Tabela 1 observou-se $t=\sum^{n} y_{i}=0$. Utilizando uma distribuição a priori não informativa $\operatorname{Beta}(1,1)$ e com base em 100 observações simuladas de $\left[\Phi^{-1}(U)\right], U$ sendo uma distribuição $\operatorname{Beta}(37,1)$, um valor aproximado de $\hat{C}_{e s} \cong 0,76$ foi obtido. Com este procedi- mento é possível estudar a variabilidade desta estimativa através de simulação. Em 50 amostras de 100 observações simuladas de

Tabela 1: Distribuição de freqüências do número de paradas de máquina (dados simulados).

\begin{tabular}{|c|c|}
\hline Número de paradas & Número de semanas \\
\hline 0 & 11 \\
\hline 1 & 17 \\
\hline 2 & 4 \\
\hline 3 & 4 \\
\hline
\end{tabular}

$U \sim \operatorname{Beta}(37,1)$, as seguintes estatísticas descritivas de $\hat{C}_{e s}$ foram calculadas:

- valor médio = 0,77568;

- mediana =0,77799;

- desvio padrão $s=0,01574$;

- $\operatorname{mínimo}=0,73758$;

- máximo $=0,81209$.

Observe que em condição estável $\gamma_{s}=P(N>4)=$ 0,0036598, o que equivale a um $C_{e s}=0,893979$. Para melhorar as estimativas podem-se observar e incorporar novos dados, recalibrando a distribuição a priori a partir da distribuição $\operatorname{Beta}(37,1)$. Veja o que ocorre com a incorporação de 12 observações de paradas semanais de máquina simuladas de uma distribuição de Poisson com média igual a 1 , especificamente: $\{0 ; 0 ; 0 ; 1 ; 1 ; 1 ; 1 ; 2 ; 2 ; 0 ; 0 ; 1\}$. Tomando como base 100 observações geradas de uma distribuição $\operatorname{Beta}(49,1)$, uma nova estimava $\hat{C}_{e s}=0,81$ foi obtida. Note que este valor é bem mais próximo de 0,89. Esse procedimento de recalibrar a incerteza pode ser repetido à medida que novos dados estejam disponíveis, permitindo que se melhore a precisão da estimativa de $C_{e s}$.

Intervalos de confiança bayesiana também podem ser facilmente obtidos. $\mathrm{O}$ problema consiste em determinar $t_{1}$ e $t_{2},\left(t_{1}<t_{2}\right)$, tais que

$$
P\left(t_{1}<C_{e s}<t_{2} \mid Y_{1}=y_{1}, \ldots, Y_{n}=y_{n}\right)=1-\gamma
$$

com $\gamma$ fixado. Utilizando uma relação entre a distribuição Beta e F de Fisher \& Snedecor, um intervalo de confiança bayesiano $\left(t_{1}, t_{2}\right)$ para $C_{e s}$ com coeficiente de confiança $1-\gamma$ é dado por

$$
\begin{aligned}
& t_{1}=\frac{1}{3} \Phi^{-1}\left(\frac{(n+b-t) f_{1}}{a+t+(n+b-t) f_{1}}\right) \\
& t_{2}=\frac{1}{3} \Phi^{-1}\left(\frac{(n+b-t) f_{2}}{a+t+(n+b-t) f_{2}}\right)
\end{aligned}
$$


onde $f_{1}$ é tal $P\left(F<f_{1}\right)=\gamma_{1}, f_{2}$ é tal $P\left(F>f_{2}\right)=\gamma_{2}, \gamma_{1+} \gamma_{2}=\gamma$ e $F \sim$ Fisher-Snedecor $[2(n+b-t), 2(a+t)]$. A demonstração deste resultado se encontra no Apêndice B.

Considere os dados da Tabela 1; $\gamma_{1}=\gamma_{2}=0,01 ; a=b=$ $1 ; n=36$ e $t=0$, os valores de $f_{1}$ e $f_{2}$ podem ser determinados: $f_{1}=0,2039 ; f_{2}=99,4856$ e o respectivo intervalo de confiança bayesiano é dado por $\left(t_{1}, t_{2}\right)=(0,3966$; 1,15283).

Um problema comumente encontrado na indústria é a demonstração de que seus processos são capazes de atender às especificações estabelecidas pelos clientes, isto é, de que os processos alcancem a capacidade desejada. A seguir um teste de capacidade sob uma perspectiva bayesiana será apresentado.

Sejam $C_{0}$ e $C_{1}, 0 \leq C_{1} \leq C_{0}$, os valores desejável e aceitável de $C_{e s}$ para um processo. Um teste de demonstração de capacidade é um procedimento estatístico definido por um $\operatorname{par}(n, d)$ de números inteiros, $d \leq n$, segundo o qual, dadas as observações $Y_{1}=y_{1}, \ldots, Y_{n}=y_{n}$, considera-se demonstrado que $C_{e s}>C_{0}$ se

$$
t=\sum_{i=1}^{n} y_{i} \leq d
$$

O par $(n, d)$ é escolhido de tal forma que

$$
\gamma=P\left(C>C_{0} \mid T>d\right)
$$

e

$$
\delta=P\left(C \leq C_{1} \mid T \leq d\right)
$$

onde $\gamma$ e $\delta$ são probabilidades especificadas. As probabilidades dadas em (7) e (8) são chamadas riscos a posteriori do produtor e do consumidor respectivamente. Para o caso particular de $\gamma_{s}$ com uma distribuição a priori não informativa $\operatorname{Beta}(1,1)$, valem os seguintes resultados:

$$
\begin{aligned}
& \gamma=\frac{(n+1) \gamma_{s 0}-\sum_{t=0}^{d}\left[1-B_{I}\left(1-\gamma_{s 0}, n-t+1, t+1\right)\right]}{n-d} \\
& \mathrm{e} \\
& \delta=\frac{\left.\sum_{t=0}^{d} B_{I}\left(1-\gamma_{s 1}, n-t+1, t+1\right)\right]}{d+1} \\
& \operatorname{com} \gamma_{s i}=1-\Phi\left(3 C_{i}\right), i=0,1 .
\end{aligned}
$$

A demonstração destes resultados encontra-se no Apêndice C. Para processos altamente capazes, existe um grande interesse em considerar testes com $d=0$. Neste caso, as equações (9) e (10) se reduzem a :

$$
\begin{aligned}
& \gamma=\frac{(n+1) \gamma_{s 0}+\left(1-\gamma_{s 0}\right)^{n+1}}{n} \\
& \mathrm{e}=\left(1-\gamma_{s 1}\right)^{n+1}
\end{aligned}
$$

O tamanho da amostra $n$ deve ser escolhido satisfazendo (11) ou (12). Note que a determinação de $n$ a partir de (11) será mais trabalhosa, ao passo que a partir de (12) pode-se determinar facilmente o número $n$, de ensaios. Especificamente,

$$
n=\frac{\log \delta}{\log \left(1-\gamma_{s 1}\right)}-1
$$

A título de ilustração, considere como valor desejável de $C_{0}, C_{1}=1$ e um risco de consumidor $\delta=0,1$. Para demonstrar que o processo apresenta este índice de capacidade, serão necessário observar conformidade em 73 ensaios. Diminuindo o risco para $\delta=0,05$ e mantendo $C_{1}=1$, deve-se observar conformidade em 2217 ensaios. Caso adote $C_{1}=1,33 \mathrm{e}$ $\delta=0,1$, deve-se ter conformidade em 69664 ensaios. Intuitivamente é natural necessitar de muitos ensaios para demonstrar que os processos são capazes de atender às especificações estabelecidas pelo cliente, principalmente quando se trata de processos de alta capacidade. Para demonstrar que um processo tem valores aceitáveis $C_{1}=2$ como valor de referência requerido em programas Seis Sigma, será necessário observar conformidade em 2.302.585.092 ensaios. Este número é coerente quando comparado com o índice tradicionalmente utilizado para processos normais e bilaterais. Para alcançar um valor de $C_{p}=2$, espera-se ter em média 0,002 itens não-conformes em um milhão de ensaios. Obviamente todos estes valores de ensaios determinados a partir (12) podem sofrer alterações, pois eles foram calculados assumindo uma distribuição $a$ priori não informativa e $d=0$.

\section{CONCLUSÕES}

A possibilidade de trabalhar com índices de capacidade baseados na fração não conforme do processo e que coincidem com os índices tradicionais quando aplicados a processos normais resolve um problema sério da aplicação desses últimos na análise de capacidade: a sua dependência da forma da distribuição da característica de qualidade $X$.

Para processo de alta capacidade, entretanto, a estimação desses índices com base no histórico de não-conformidades do processo esbarra no problema da necessidade de esti- 
mar com base em amostras em que não há não-conformidades. A inferência bayesiana nesta situação permite-nos tirar conclusões racionais através de procedimentos bastante simples, isto é, envolvendo apenas um mecanismo para simular observações de uma distribuição Beta. Além disto, por ser esta distribuição conjugada para a amostragem binomial, estas estimativas podem ser recalibradas periodicamente à medida que novos dados se tornem disponíveis.

A escolha de outras distribuições além da Beta como distribuição a priori é certamente uma outra alternativa a ser empregada. No entanto, a propriedade de distribuição conjugada passa a não valer, o que viria dificultar a obtenção da distribuição a posteriori, requerendo maiores esforços computacionais.

Atualmente existe uma expressiva tendência de as empresas adotarem o programa Seis Sigma com o objetivo de melhorar a produtividade de seus processos. No entanto, deve-se salientar que para tais processos alcançarem os valores de referência como $C_{e s}=1,5$ ou $C_{e s}=2$, conforme exige o programa Seis Sigma, demandará das empresas recursos monetários e de disponibilidade de tempo que nem sempre são facilmente acessíveis.

\section{- Agradecimentos}

Os autores agradecem as valiosas sugestões dadas pelos anônimos avaliadores.

\section{APÊNDICE}

\section{Apêndice A Distribuição a priori e a posteriori de $C_{e s}$}

As proposições (A.1) e (A.2\}) têm importância fundamental na inferência Bayesiana para o índice de capacidade unilateral superior $C_{e s}$.

Proposição A.1 Se $\gamma_{\mathrm{s}}$ tem distribuição a priori Beta(a,b), então a distribuição a priori induzida para $\mathrm{C}_{\mathrm{es}}$ é dada por:

$H(\theta)=1-B_{I}[(1-\Phi(3 \theta)), a, b],-\infty<\theta<\infty$

que é absolutamente contínua com função de densidade

$$
h(\theta)=\frac{3 \phi(3 \theta)}{B(a, b)}[\Phi(3 \theta)]^{(b-1)}[1-\Phi(3 \theta)]^{(a-1)},-\infty<\theta \infty
$$

onde $B_{I}(x, a, b)$ denota $P(U \leq x), U \sim \operatorname{Beta}(a, b)$ e $\phi($.$) é a função de densidade da distribuição normal padrão.$

Prova: Como $\gamma_{\mathrm{s}}$ tem distribuição $\operatorname{Beta}(a, b)$

$$
\begin{aligned}
P\left(C_{e s} \leq \theta\right) & =P\left(\frac{1}{3} \Phi^{-1}\left(1-\gamma_{s}\right) \leq \theta\right) \\
& =1-\int_{0}^{1-\Phi(3 \theta)} \frac{\Gamma(a+b)}{\Gamma(a) \Gamma(b)} \gamma_{u}^{a-1}\left(1-\gamma_{u}\right)^{b-1} \\
& =1-B_{I}[1-\Phi(3 \theta), a, b]
\end{aligned}
$$


Proposição A.2 A distribuição a posteriori de $\mathrm{C}_{\mathrm{es}}$ dado $\mathrm{Y}_{1}=\mathrm{y}_{1}, \ldots, \mathrm{Y}_{\mathrm{n}}=\mathrm{y}_{\mathrm{n}}$ tem função densidade

$h\left(\theta \mid y_{1}, \ldots, y_{n}\right)=\frac{3 \phi(3 \theta)[\Phi(3 \theta)]^{n+b-t-1}[1-\Phi(3 \theta)]^{a+t-1}}{B(n+b-t, a+t)}$

onde $t=\sum_{i=1}^{n} y_{i}$

Prova: A função de verossimilhança de $\theta$ dada a amostra observada $\mathrm{y}_{1}, \ldots, \mathrm{y}_{\mathrm{n}}$ satisfaz

$L(\theta) \propto \gamma_{s}^{t}\left(1-\gamma_{s}\right)^{n-t}$

onde $\gamma_{s}=1-\Phi(3 \theta)$. Considerando (A.3), e a função densidade a priori dada na proposição (A.1), pode-se mostrar que a função densidade a posteriori de $\theta$ é dada por:

$h\left(\theta \mid y_{1}, \ldots, y_{n}\right)=k \frac{3 \phi(3 \theta)}{B(a, b)}[1-\Phi(3 \theta)]^{a+t-1}[1-\Phi(3 \theta)]^{n-t+b-1}$

onde

$k^{-1}=\int_{-\infty}^{\infty} \frac{3 \phi(3 \theta)}{B(a, b)}[1-\Phi(3 \theta)]^{a+t-1}[\Phi(3 \theta)]^{n-t+b-1} d \theta$

Fazendo $u=1-[1-\Phi(3 \theta)]$ a integral acima se reduz a

$k^{-1}=\int_{0}^{1}[1-u]^{a+t-1}[u]^{n-t+b-1} d u=B(n+b-t, a+t)$

Da proposição (A.2) resulta o seguinte corolário.

Corolário A.1 O estimador de Bayes de $C_{e s}$ é dado por:

$\hat{C}_{e s}=\frac{E\left[\Phi^{-1}(U)\right]}{3}$

em que $U \sim \operatorname{Beta}(n+b-t, a+t)$.

Prova: Sob perda quadrática o estimador de Bayes de $C_{e s}$ é dado por:

$\hat{C}_{e s}=\int_{-\infty}^{\infty} \theta \pi\left(\theta \mid y_{1}, \ldots, y_{n}\right) d \theta$

em que $h\left(\theta \mid y_{1}, \ldots, y_{n}\right)$ é a densidade da distribuição a posteriori de $C_{e s}$ da proposição (A.2). Fazendo a mudança de variáveis $u=1-[1-\Phi(3 \theta)]$ a integral acima.

$\hat{C}_{e s}=\frac{\int_{0}^{1} \frac{1}{3} \Phi^{-1}(u) u^{n+b-t-1}(1-u)^{a+t-1} d u}{B(n+b-t, a+t)}=\frac{1}{3} E\left[\Phi^{-1}(U)\right]$ 
Apêndice B Intervalo de confiança bayesiano para $C_{e s}$

O problema consiste em determinar $t_{1}$ e $t_{2},\left(t_{1}<t_{2}\right)$ tais que

$P\left(t_{1}<C_{e s}<t_{2} \mid Y_{1}=y_{1}, \ldots, Y_{n}=y_{n}\right)=1-\gamma$

com $\gamma$ fixado. Isto é equivalente a

$P\left(C_{e s}<t_{1}\right)=\int_{-\infty}^{t_{1}} h\left(\theta \mid Y_{1}=y_{1}, \ldots, Y_{n}=y_{n}\right) d \theta=\gamma$

e

$P\left(C_{e s}>t_{2}\right)=\int_{t_{2}}^{\infty} h\left(\theta \mid Y_{1}=y_{1}, \ldots, Y_{n}=y_{n}\right) d \theta=\gamma$

em que $h\left(\theta \mid Y_{1}=y_{1}, \ldots, Y_{n}=y_{n}\right)$ é a distribuição a posteriori de $C_{e s}$ dada em (A.2) e $\gamma_{1}+\gamma_{2}=\gamma$. Quando $\gamma_{1}=\gamma_{2}=\gamma / 2,\left(t_{1}, t_{2}\right)$ é um intervalo de confiança bayesiano simétrico para $C_{e s}$.

Proposição B.1 O intervalo de confiança bayesiano $\left(t_{1}, t_{2}\right)$ para $C_{e s}$ com coeficiente de confiança 1- $\gamma$ é dado por $t_{1}=\frac{1}{3} \Phi^{-1}\left(\frac{(n+b-t) f_{1}}{a+t+(n+b-t) f_{1}}\right)$

e

$t_{2}=\frac{1}{3} \Phi^{-1}\left(\frac{(n+b-t) f_{2}}{a+t+(n+b-t) f_{2}}\right)$

onde $f_{1}$ é tal $P\left(F<f_{1}\right)=\gamma_{1}, f_{2}$ é tal $\left.P F>f_{2}\right)=\gamma_{2}, \gamma_{1+} \gamma_{2}=\gamma$ e $F \sim F i$ sher-Snedecor $[2(n+b-t), 2(a+t)]$.

Prova: Fazendo a mudança de variáveis $u=1-[1-\Phi(3 \theta)]$ em (A.2) determinam-se $t_{1}$ e $t_{2}$ de (B.1) e (B.2) tais que

$$
P\left(C_{e s}<t_{1}\right)-P\left(U<\Phi\left(3 t_{1}\right)\right)=\int_{0}^{\Phi\left(3 t_{1}\right)} \frac{u^{n+b-t-1}(1-u)^{a+t-1}}{B(n+b-t, a+t)} d u=\gamma_{1}
$$

e

$$
P\left(C_{e s}>t_{2}\right)-P\left(U>\Phi\left(3 t_{2}\right)\right)=\int_{\Phi\left(3 t_{2}\right)}^{1} \frac{u^{n+b-t-1}(1-u)^{a+t-1}}{B(n+b-t, a+t)} d u=\gamma
$$

em que $U \sim \operatorname{Beta}(n+b-t, a+t)$. Para resolver as equações acima em $t_{1}$ e $t_{2}$, a seguinte relação entre as distribuições Beta e F de Fisher-Snedecor foi empregada. Se $W \sim \operatorname{Beta}(p, q)$ e $F \sim F(2 p, 2 q)$ então:

$$
P(W<w)=P\left(\frac{p F / q}{1+p F / q}<w\right)=P\left(F<\frac{q w}{p(1-w)}\right)
$$


Como $U \sim \operatorname{Beta}(n+b-t, a+t)$ segue-se de (B.3) que

$$
P\left(U<\Phi\left(3 t_{1}\right)\right)=P\left(F<\frac{(a+t) \Phi\left(3 t_{1}\right)}{(n+b-t)\left(1-\Phi\left(3 t_{1}\right)\right)}\right)=\gamma_{1}
$$

onde F Fisher-Snedecor [2(n+b-t), 2(a+t)]. Conseqüentemente, temos

$$
f_{1}=\frac{(a+t) \Phi\left(3 t_{1}\right)}{(n+b-t)\left(1-\Phi\left(3 t_{1}\right)\right)}
$$

onde $f_{1}$ é tal que $P\left(F<f_{1}\right)$. Resolvendo (B.4) em $t_{1}$, obtém-se

$$
t_{1}=\frac{1}{3} \Phi^{-1}\left(\frac{(n+b-t) f_{1}}{a+t+(n+b-t) f_{1}}\right)
$$

Similarmente, temos

$$
P\left(U>\Phi\left(3 t_{2}\right)\right)=P\left(F<\frac{(a+t) \Phi\left(3 t_{2}\right)}{(n+b-t)\left(1-\Phi\left(3 t_{2}\right)\right)}\right)=\gamma_{2}
$$

$\mathrm{e}$

$$
f_{2}=\frac{(a+t) \Phi\left(3 t_{2}\right)}{(n+b-t)\left(1-\Phi\left(3 t_{2}\right)\right)}
$$

onde $f_{2}$ é tal que $P\left(F>f_{2}\right)$. Resolvendo esta última equação em $t_{2}$, obtém-se

$$
t_{2}=\frac{1}{3} \Phi^{-1}\left(\frac{(n+b-t) f_{2}}{a+t+(n+b-t) f_{2}}\right)
$$

Apêndice C Teste de demonstração de capacidade

Proposição C.1 Para valores fixados de $\gamma$ e $\theta$, o par $(n, d)$ satisfaz as seguintes equações:

$$
\gamma=\frac{(n+1) \gamma_{s 0}-\sum_{t=0}^{d}\left[1-B_{I}\left(1-\gamma_{s 0}, n-t+1, t+1\right)\right]}{n-d}
$$

e

$$
\delta=\frac{\sum_{t=0}^{d} B_{I}\left(1-\gamma_{s 1}, n-t+1, t+1\right)}{d+1}
$$

$\operatorname{com} \gamma_{s i}=1-\Phi\left(3 C_{i}\right), i=0,1$ 
Barriga; Ho \& Borges

Prova: A probabilidade dada em (7) pode ser expressa como

$$
\begin{aligned}
\gamma & =\frac{P\left(C_{e s}>C_{0}, T>D\right)}{P(T>d)} \\
& =\frac{\int_{C_{0}}^{\infty}\left[1-P\left(T \leq d \mid C_{e s}=v\right)\right] \pi(v) d v}{\int_{-\infty}^{\infty}\left[1-P\left(T \leq d \mid C_{e s}=v\right)\right] \pi(v) d v} \\
& =\frac{\int_{C_{0}}^{\infty}\left[1-\sum_{t=0}^{d}(n) \gamma_{s}^{t}\left(1-\gamma_{s}\right)^{n-t}\right] \pi(v) d v}{\int_{-\infty}^{\infty}\left[1-\sum_{t=0}^{d}(n) \gamma_{s}^{t}\left(1-\gamma_{s}\right)^{n-t}\right] \pi(v) d v}
\end{aligned}
$$

em que $\gamma_{s}=1-\Phi\left(3 C_{e s}\right)=1-\Phi(3 v)$ e $\pi(v)=3 \phi(3 v)$ é a priori induzida dada na proposição A.1. Fazendo a mudança de variáveis $\mathrm{u}=\Phi(3 v)$, a expressão acima se reduz a

$$
\begin{aligned}
\gamma=\frac{\gamma_{s 0}-\sum_{t=0}^{d}\left(\begin{array}{l}
n \\
t
\end{array}\right) \int_{C_{0}}^{\infty}[1-\Phi(3 v)]^{t}[\Phi(3 v)]^{n-t} 3 \phi(3 v) d v}{1-\sum_{t=0}^{d}\left(\begin{array}{l}
n \\
t
\end{array}\right)_{-\infty}^{\infty}[1-\Phi(3 v)]^{t}[\Phi(3 v)]^{n-t} 3 \phi(3 v) d v} \\
=\frac{\gamma_{s 0}-\sum_{t=0}^{d}\left(\begin{array}{l}
n \\
t
\end{array}\right) B(n-t+1, t+1)\left[1-B_{I}\left(1-\gamma_{s 0}, n-t+1, t+1\right)\right]}{1-\sum_{t=0}^{d}(n) B(n-t+1, t+1)} \\
=\frac{(n+1) \gamma_{s 0}-\sum_{t=0}^{d}\left[1-B_{I}\left(1-\gamma_{s 0}, n-t+1, t+1\right)\right]}{n-d}
\end{aligned}
$$

Analogamente,

$$
\begin{aligned}
\delta & =\frac{P\left(C_{e s} \leq C_{1}, T \leq D\right)}{P(T \leq d)} \\
& =\frac{\int_{-\infty}^{C_{1}} P\left(T \leq d \mid C_{e s}=v\right) \pi(v) d v}{\int_{-\infty}^{\infty} P\left(T \leq d \mid C_{e s}=v\right) \pi(v) d v}
\end{aligned}
$$

48

Revista Produção v. 13 n. 12003 


$$
\begin{aligned}
= & \frac{\int_{-\infty}^{C_{1}}\left[\sum_{t=0}^{d}\left(\begin{array}{l}
n \\
c
\end{array}\right) \gamma_{s}^{t}\left(1-\gamma_{s}\right)^{n-t}\right] \pi(v) d v}{\int_{-\infty}^{\infty}\left[\begin{array}{l}
d \\
\left.\sum_{t=0}\left(\begin{array}{l}
n \\
c
\end{array}\right) \gamma_{s}^{t}\left(1-\gamma_{s}\right)^{n-t}\right] \pi(v) d v
\end{array}\right.} \\
= & \frac{\sum_{t=0}^{d}\left(\begin{array}{l}
n \\
t
\end{array}\right) B(n-t+1, t+1)\left[B_{I}\left(1-\gamma_{s 1}, n-t+1, t+1\right)\right]}{\sum_{t=0}^{d}\left(\begin{array}{l}
n \\
t
\end{array}\right) B(n-t+1, t+1)} \\
= & \frac{\sum_{t=0}^{d}\left[B_{I}\left(1-\gamma_{s 1}, n-t+1, t+1\right)\right]}{d+1}
\end{aligned}
$$

\section{Artigo recebido em 1/08/2002 \\ Revisado em 17/12/2002 \\ Aprovado para publicação em 21/02/2003}

- Referências

BERNARDO, J.M \& IRONY, T. Z. (1996). A general multivariate Bayesian process capability index. The Statistician, 45(3) 487-502.

BISSELL, A. F., (1990). How reliable is your capability index?. Applied Statistics, 39(3), 331-340.

BORGES, W. S. \& Ho, L. L. (2000). On the sampling distribution of Clement's capability index. Communication in Statistics - Simulation and Computation, 29(1), 121-38.

BORGES W. \& Ho L. L. (2001). A fraction defective based capability index. Quality and Reliability Engineering International, 17, 447-58

BOTHE, D. R. (1999). A capability index for multiple process streams. Quality Engineering, 11(4), 613-8.
BOYLES, R. A. (1991). The Taguchi capability index. Journal of Quality Technology, 23(1), 17-26.

CHENG, S. W.(1994-95). Practical implementation of the process capability indices. Quality Engineering, 7(2), 239-59.

CHENG, S. W. \& SPIRING, F. A. (1989). Assessing process capability: a Bayesian approach, IIE Transaction. 21, 97-8.

CHOI, K. C.; NAM, K. H. \& PARK, D. H. (1996), Estimation of capability index based on bootstrap method, Microelectron. Reliabil. 36(9), 1141-53.

CHOU, Y.M. \& OWEN, D. B. (1989). On the distribution of the estimated process capability indices. Communs. Statist. Theory Meth., 18, 4549-60.
GUPTA, A. K \& KOTZ, S. (1997). A new process capability index. Metrika, 45, 213-24.

JOHNSON, T. (1992). The relationship of C_ $\{\mathrm{pm}\}$ to square error loss, Journal of Quality Technology, 24(4), 211-5.

KAMINSKY, F. C.; DOVICH, R. A. \& BURKE, R. J. (1998). Process capability indices: now and in the future. Quality Engineering, 10(3), 445-53.

KANE, V. E. (1986). Process capability indices, Journal of Quality Technology 18(1), 41-52.

KOTZ, S., JOHNSON, N.L. (1993). Process Capability Indices, Chapman and Hall, London.
KOTZ, S., PEARN, W. L, \& JOHNSON, C.C. (1993). Some process capability indices are more reliable than one might think Applied statistics, 42(1), 56-62.

PEARN, W. L. \& CHEN, K. S. (1997) Capability indices for non-normal distributions with an application in Electrolytic Capacitor Manufacturing Microelectron. Reliab., 37(12), 1853-8.

PEARN, W. L, \& KOTZ, S. (1994). Application of Clement's method for calculating second and third generation process capability indices for nonnormal Pearsonian populations. Quality Engineering, 7(1), 139-45.

PEARN, W. L., KOTZ, S. \& JOHNSON C.C. (1992). Distributions and inferential properties of process capability indices Journal of Quality Technology, 24(4) 216-33. 